\title{
Research on the risk of Shanghai Composite Index based on VaR and GARCH model
}

\author{
Bing Yang ${ }^{1, a}$, Chenggang $\mathrm{LI}^{2, \mathrm{~b}^{*}}$, Di Wang ${ }^{1, \mathrm{c}}$ and Xin $\mathrm{He}^{1, \mathrm{~d}}$ \\ ${ }^{1}$ Faculty of Finance, Guizhou University of Finance and Economics, Guiyang 550025, China ; \\ ${ }^{2}$ Faculty of Science, National University of Singapore, 117546, Singapore.

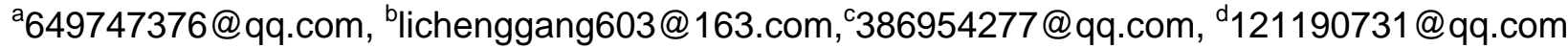

\begin{abstract}
Analyzing the risk of Shanghai Composite Index is beneficial to investors in stock investment, and provides reference for investors. This paper selects the daily data of Shanghai Composite Index from January 1, 2005 to December 30, 2016 as the research object, and uses VaR and GARCH model to empirically research the risk of Shanghai Composite Index. The results show that, through the fitting of different GARCH models, the EGARCH(1,1) model under the $t$ distribution can get the best fitting effect. Compared VaR measuring value and the failure probability of the inspection under different distribution, it can be found that the VaR measuring value under the t distribution has smaller volatility, higher accuracy, and minimal failure probability.
\end{abstract}

Keywords: Shanghai Composite Index, VaR, GARCH model.

\section{Introduction}

The sample stocks of the Shanghai Composite Index are all listed shares, including A shares and B shares. They reflect the price changes of the listing stocks in the Shanghai Stock Exchange. Stock price reflects the development of the list company, and the Shanghai Composite Index reflects the overall economy level of China's overall entity. The rise and fall of a country's economy can be reflected by the ups and downs of the stock price. the trend of the Shanghai Composite Index is the most intuitive expression of the overall economy development. Therefore, the forecast of the Shanghai Composite Index is of great significance to the regulation of the state in the real economy and the formulation of regional policies.

Model setting

\section{Calculation method of VaR}

In 1994, Morgan Company put forward Value at Risk, which is commonly used in the risk management of financial institutions. VaR means Value at Risk. In other words, it means the maximum possible loss of a financial asset or portfolio of securities under normal market volatility ${ }^{[1]}$. More precisely, it means the maximum possible loss of a financial asset or portfolio at a certain probability level (confidence level) for a particular period of time. It can be expressed as the following formula:

$$
P\left(\Delta \mathrm{p}^{*} \Delta \mathrm{t} \leq \mathrm{VaR}\right)=\mathrm{a}
$$

$P$ represents that the loss of asset value is less than the probability of maximum loss. $\Delta p$ represents the amount of a financial asset in a certain period $t$ of loss of value. VaR represents the value at the level of confidence $a$, ie, the upper limit of the loss; a indicates a given confidence level.

\section{GARCH model}

Bollerslev (1986) ${ }^{[3]}$ proposed a generalized autoregressive conditional heteroskedasticity model based on the $A R C H$ model, which is called GARCH model. GARCH model is an important expansion of $A R C H$ model. ARCH model is a special case of $G A R C H$ model. GARCH model can be expressed as: 


$$
\begin{aligned}
& \mathrm{r}_{t}=c_{0}+\sum_{i=1}^{m} c_{i} r_{t-i}+a_{t}-\sum_{j=1}^{n} \theta_{j} a_{t-j}, a=\delta_{t} \varepsilon_{t} \\
& \delta_{\mathrm{t}}^{2}=\omega+\sum_{i=1}^{p} \alpha_{i} a_{t-i}+\sum_{j=1}^{q} \beta_{j} \delta_{t-j}^{2}
\end{aligned}
$$

Among them, ${ }^{\varepsilon_{\mathrm{t}}}$ in formula (2) is subject to the standard normal distribution. $\mathrm{r}_{\mathrm{t}}$ is subject to $\operatorname{ARMA}(m, n)$ model. ${ }^{\delta_{\mathrm{t}}^{2}}$ in formula (3) is subject to $\operatorname{GARCH}(p, q)$ model.

\section{An Empirical Analysis of Shanghai Stock Index}

\subsection{Data Sources}

This paper selects the closing price of daily data of Shanghai Composite Index from January 1, 2005 to December 30, 2016, a total of 2913 data as the sample. The data from the website of Shanghai Securities News (http://szb.dooland.com/). We constructs the sequence $\{\mathrm{p}\}$, then constructs the logarithmic return sequence $\{r\}$, establishes the conditional heteroscedasticity model for the sequence $\{r\}$, and studies the fluctuation of return ${ }^{[4]}$.

The time series chart of the return $\{r\}$ is given as the time series of the log return $\{r\}$.From the linear graph of the logarithmic return sequence $\{r\}$ of the Shanghai Composite Index, it can be observed that the fluctuation of the logarithmic return has a cluster phenomenon.

\subsection{Descriptive statistical analysis}

Descriptive statistical analysis of return data is the mean return of Shanghai Composite Index from January 1, 2005 to December 30, 2016 is 0.000314 . The median is 0.000936 . The maximum and minimum are 0.090343 and -0.092562 . The skewness value is -0.552122 , which is less than zero. So you can see that, from January 1, 2005 to December 30, 2016, the Shanghai Composite Index is a left trailing shape. It indicates that the Shanghai Composite Index in the early period is from the more and more stable to more and more steep. The Kurtosis value is 6.886250 , which is greater than 3 . It indicates that the distribution pattern of Shanghai Composite Index from January 1, 2005 to December 30, 2016 is greater than the normal distribution. It shows that the return sequence has the characteristics of spikes and trailing edges. The Jarque-Bera statistic is 198.438 and the value is 0.00000 . So the assumption that the logarithmic return is subject to the normal distribution is rejected.

\subsection{Stability test and autocorrelation test}

We use ADF test to tested sequence stability ${ }^{[5]}$, and the results are shown in Table 1.

\begin{tabular}{|c|c|c|c|}
\hline & & t- statistic & Prob. \\
\hline \multicolumn{2}{|c|}{ Augmented Dickey-Fuller test statistic } & -52.71719 & 0.0001 \\
\hline \multirow{3}{*}{ Test critical values; } & $1 \%$ level & -3.432406 & \\
\hline & $5 \%$ level & -2.862334 & \\
\hline & $10 \%$ level & -2.567237 & \\
\hline
\end{tabular}

Table 1 Stability test of Shanghai Composite Index

It can be seen from Table 1 that, the ADF test value of sequence $\{r\}$ is less than -52.71719 , and is less than its critical value at the significance level of $1 \%, 5 \%$ and $10 \%$. Therefore, the time series can be considered to be stationary.

The results of autocorrelation and partial autocorrelation tests of return sequence we can see that the autocorrelation coefficient of the sequence falls into the random interval. There is no sequence correlation. The autocorrelation and partial autocorrelation values are close to zero in all order. All the statistics are not significant. The corresponding values of the statistic were significantly greater than 0.05 . So the sequence has no significant autocorrelation at the $5 \%$ significance level.

\subsection{Build the GARCH model}

In this paper, we select the GARCH $(1,1)$ model. Based on the GARCH amily model of the logarithmic return residual series under different distributions, we determine that the fitness effect of which distribution of $\operatorname{GARCH}(1,1)$ is the best. We compare Akaike info criterion and Schwarz 
criterion. The results are shown in Table 2. As can be seen from Table 2, the logarithmic function of the TGARCH(1,1) model under t distribution has the largest value and the Akaike info criterion and Schwarz criterion are relatively small. Therefore, the fitness of the EGARCH(1,1) model under $t$ distribution is optimal.

\subsection{Calculate the VaR value}

Table 2 Logarithmic return residuals under different parameter models

\begin{tabular}{|c|c|c|c|}
\hline \multirow{2}{*}{ Model parameters } & \multicolumn{3}{|c|}{ GARCH(1,1) model } \\
\cline { 2 - 4 } & Normal distribution & T distribution & GED distribution \\
\hline Akaike info criterion & -5.435211 & -5.488704 & -5.487644 \\
\hline Schwarz criterion & -5.429054 & -5.480495 & -5.479435 \\
\hline Log Likelihood & 7916.668 & 7925.553 & 7994.01 \\
\hline \multirow{2}{*}{ Model parameters } & \multicolumn{3}{|c|}{ TGARCH(1,1) model } \\
\cline { 2 - 4 } & Normal distribution & T distribution & GED distribution \\
\hline Akaike info criterion & -5.434761 & -5.488017 & -5.486992 \\
\hline Schwarz criterion & -5.426552 & -5.477755 & -5.47673 \\
\hline Log Likelihood & 7917.013 & 7995.553 & 7994.06 \\
\hline \multirow{2}{*}{ Model parameters } & EGARCH(1,1) model \\
\cline { 2 - 4 } & Normal distribution & T distribution & GED distribution \\
\hline Akaike info criterion & -5.439935 & -5.49122 & -5.490469 \\
\hline Schwarz criterion & -5.431735 & -5.480958 & -5.480207 \\
\hline Log Likelihood & 7924.545 & 7983.216 & 7992.124 \\
\hline
\end{tabular}

By returning the GARCH model family of different distributions and returning the selected model back to the original model, the VaR statistics of the minimum, maximum, mean, and standard deviation are calculated. Compared the VaR statistics of the GARCH model family under different distributions, the results are shown in Table 3.

Table 3 VaR statistical tables of different models

\begin{tabular}{|c|c|c|c|c|c|c|}
\hline \multirow{2}{*}{ Distribution } & \multirow{2}{*}{ Model } & \multicolumn{5}{|c|}{ VaR } \\
\cline { 2 - 6 } & & Min & Max & Mean & St.d-d & Median \\
\hline \multirow{2}{*}{$\begin{array}{c}\text { Normal } \\
\text { distribution }\end{array}$} & GARCH & -6.665763 & 4.610679 & 0.003861 & 1.000945 & 0.033977 \\
\cline { 2 - 7 } & TARCH & -6.666772 & 4.567154 & 0.004234 & 1.000874 & 0.033967 \\
\cline { 2 - 6 } & $E A R C H$ & -6.896415 & 4.258901 & 0.003759 & 1.000746 & 0.033634 \\
\hline \multirow{2}{*}{$\begin{array}{c}\mathrm{T} \\
\text { distribution }\end{array}$} & GARCH & -6.579423 & 4.597964 & 0.004054 & 0.993839 & 0.033700 \\
\cline { 2 - 7 } & $T A R C H$ & -6.579458 & 4.597687 & 0.004065 & 0.993837 & 0.033699 \\
\cline { 2 - 7 } $\begin{array}{c}\text { GED } \\
\text { distribution }\end{array}$ & EARCH & -6.824685 & 4.269967 & 0.003724 & 0.994046 & 0.033027 \\
\cline { 2 - 7 } & GARCH & -6.668179 & 4.637304 & 0.003995 & 1.004385 & 0.034077 \\
\cline { 2 - 7 } & EARCH & -6.670193 & 4.616089 & 0.004175 & 1.004322 & 0.034069 \\
\hline \multirow{2}{*}{ It } & -6.904643 & 4.294915 & 0.003787 & 1.004051 & 0.033533 \\
\hline
\end{tabular}

It can be seen from Table 3 that, by comparing the measured VaR at the same confidence level under different distributions, the maximum, minimum, mean and median are no significant differences.The above statistical results show that, at the same significance level and under three distributions, the standard deviation of the $t$ distribution fluctuates around 0.938 , which is the smallest. In the T distribution, the standard deviation of TGARCH model in the three models is 0.993837, which is minimum. Therefore, we can draw the following conclusions: under the $t$ distribution, the three types of GARCH models can better describe the thick tail characteristics of the return series, and can give more accurate VaR values. However, the TGARCH model is optimal.

Compared with the probability of success and failure of different models at the same confidence level, the results are shown in Table 4. We compare the failure frequency of the VaR-GARCH model, TGARCH model and VaR-EARCH model at the same confidence level. At the 95\% confidence level, and the total 500 observation days, the number of expected failure days is 30 days. But the actual results show that under different GARCH family models, the failure probability of each model is 
different. The failure probability of VaR-TARCH model is the smallest, the failure probability of the VaR-EARCH model is the largest, and the failure probability of the VaR-GARCH model is in the center. Therefore, we can conclude that, the results obtained using the VaR-TARCH model are more accurate than the VaR-GARCH model and VaR-EARCH model.

Table 4 Failure probability of GARCH model family

\begin{tabular}{|c|c|c|c|c|}
\hline \multicolumn{5}{|c|}{ The failure probability test of the VaR-GARCH model } \\
\hline Inspection days & Confidence & Expected failure days & Actual failure days & Failure probability \\
\hline 500 & $95 \%$ & 30 & 33 & $6.6 \%$ \\
\hline \multicolumn{5}{|c|}{ The failure probability test of the VaR-TARCH model } \\
\hline Inspection days & Confidence & Expected failure days & Actual failure days & Failure probability \\
\hline 500 & $95 \%$ & 30 & 30 & $6.0 \%$ \\
\hline \multicolumn{7}{|c|}{ The failure probability test of the VaR-EARCH model } \\
\hline Inspection days & Confidence & Expected failure days & Actual failure days & Failure probability \\
\hline 500 & $95 \%$ & 30 & 35 & $7.0 \%$ \\
\hline
\end{tabular}

\section{Conclusions}

This paper does stationary test, autocorrelation test and ARCH effect test of Shanghai Composite Index. The results show that the logarithmic return series of the Shanghai Composite Index is a spike and thick tail, and the distribution is stable. There is ARCH effect. For the risk judgment of the Shanghai Composite Index, this paper uses the corresponding GARCH family model and VaR to analyze the risk. The results show that, through the fitting of different GARCH models, the EGARCH(1,1) model under the $t$ distribution can get the best fitting effect. Compared VaR measuring value and the failure probability of the inspection under different distribution, it can be found that the VaR measuring value under the $t$ distribution has smaller volatility, higher accuracy, and minimal failure probability.

\section{Acknowledgments}

This work was financially supported by he National Scholarship Foundation.

\section{References}

[1] Z.L. Zheng, R. Chen, Financail engineering, forth edition), Beijing, Higher Education Press, 2016.

[2] Engle.R. F,Autoregressive conditional heteroskedasticity with estimates of the variance of U.K. Inflation. Econometrica.4(1982) 987-1008.

[3] Bollerslev T. Generalized autoregressive conditional heteroskedasticity. Journal of Econometrics.31(1986) 307-327.

[4] Xu. Q. F, Jiang C X. R software and its application in financial quantity analysis. Beijing: Tinghua University Press (2015).

[5] Gao.T.M,Econometric economic ananlysis method and modelling - Eviews application and its examples (Second Edition). Beijing: Tinghua University Press(2009). 


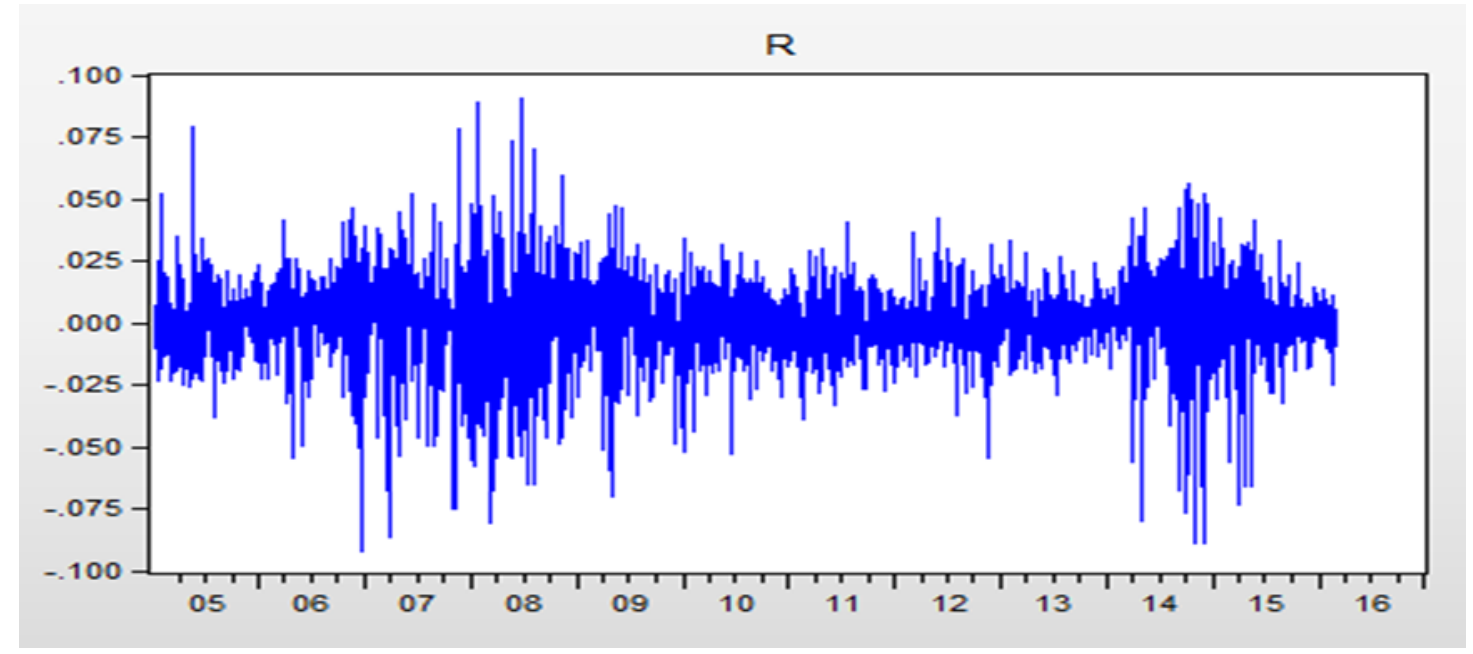

